\title{
Effect of an Internal Impact Balance Mechanism on the Perceptible Recoil of Machine Gun
}

\author{
Liu Qiang ${ }^{1}$, Zhou Kedong ${ }^{1, *}$, Shen Chao ${ }^{1}$, He lei ${ }^{1}$ \\ ${ }^{1}$ School of Mechanical Engineering, Nanjing University of Science and Technology, Nanjing 210094, China
}

\begin{abstract}
In order to reasonably reduce the perceptible recoil of firearms, and design a recoil reduction device that can be commonly used in automatic rifles, semi-automatic rifles, and machine guns, a balancing mechanism for recoil reduction technology was proposed in this paper. With the perceptible recoil generated by the impact from automat to the balance mechanism as the objective function, the parametric analysis of the mass of the balance weight and the spring stiffness of the balance mechanism was conducted. The analysis showed that the influence of the mass of the balance weight on the reduction of the perceptible recoil of the balance mechanism is obviously greater than that of the spring stiffness of the balance mechanism within the allowable ranges of the two factors. The research results provide a reference for gun recoil reduction technology.
\end{abstract}

\section{Introduction}

The problem of recoil of firearms has always been a hot issue in the design of firearms. Reduction of the perceptible recoil of firearms during shooting, can not only reduce the shooter's fatigue, but also help to improve the shooting accuracy of firearms. It is a topic of great research significance. In this paper, recoil force refers to the impact force when the bolt carrier hits the gun tail, and the perceptible recoil refers to the output pressure of the pressure sensor contacting with the gun butt during the shooting process of the gun.

Compared with other guns, the machine gun has more mechanism actions in the recoil process. In order to make these mechanism actions reliable, when the bolt carrier recoils in place, the kinetic energy is often surplus. Therefore when the bolt carrier hits the tail of the gun, it will produce greater recoil than other firearms. The traditional machine gun uses a buffer spring (M249 machine gun, etc.), friction buffer (M60 machine gun, etc.) to buffer machine guns, to reduce the sensible recoil of the machine gun. But often because of the high stiffness of the buffer itself, the force of the bolt assembly impacting the buffer can still be transmitted to the shooter through the tail and butt of the gun, forming a significant sensible recoil.

The Russian AK109 rifle first used the automatic balance method. That is, a long balance weight is added above the bolt carrier, and the bottom of the balance block and the top of the bolt carrier have racks with the same tooth shape, the connection between the two depends on a six-tooth gear fixed on the receiver to ensure that the bolt carrier is driven backward, through the six-tooth gear transmission, while the balance weight moves, to ensures that the bolt carrier and the balance mechanism reach the dead point position at the same time, and the impact force on the tail of the bolt carrier at its extreme rear end position is reduced .

Although the automatic balance method of the AK109 rifle can reduce recoil, it still has many shortcomings.

1) It still cannot avoid the direct collision between the bolt carrier and the gun tail;

2) The use of "gear", a more precise mechanical device in firearms reduces the reliability of firearms;

3) The balance mechanism can only be arranged above the bolt carrier. This arrangement is suitable for rifles. When used in a machine gun, it will interfere with the feed mechanism.

Therefore, a new type of internal collision balance mechanism is proposed to improve the above three shortcomings.

\section{Structure design of balance mechanism}

\subsection{Structure of balance mechanism}

FIG. 1 shows the overall structure of the balance mechanism. As shown in the figure, the balance mechanism is composed of a bolt carrier, a balance weight, a return spring, a gun tail, a balancing mechanism spring, a stop block, a locking plate, and a release guide. Among them, the lock plate and the balance weight are

* Corresponding author: zkd81151@ njust.edu.cn 
connected together by a leaf spring, so that the lock plate can only move up and down relative to the balance weight. Figure 2 is the assembly diagram of balance weight and lock block. The tail of the gun and the stop block are fixedly connected with the receiver, and the stopper gives the front limit position of the balance block moving in the direction of the muzzle.

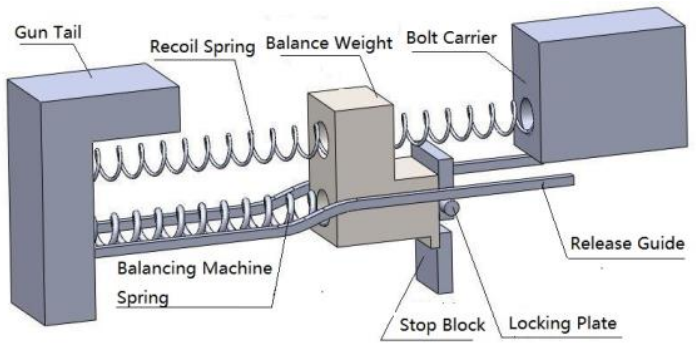

Figure 1. Overall structure of balance mechanism

Because of the effect of the leaf spring in FIG. 2, when the lock block is not subjected to other external forces, the position relative to the balance weight in FIG. 1 and FIG. 2 will be maintained. Balance weight has a through hole through which the recoil spring passes, and does not interfere with the balance weight. There is a blind hole in the lower half of the back plane of balance weight, and the balance mechanism spring is connected internally. The tail ends of the balance mechanism spring and the recoil spring are fixed on the tail of the gun. In addition, in order to avoid the bolt carrier, balance block and gun tail contacting and squeezing each other in turn, in this case, it is equivalent to the bolt carrier and the balance weight hitting the tail of the gun at the same time. The impact mass is very large, which will cause a large sensible recoil force. When the bolt carrier hits the gun tail, the balance weight oscillates back and forth in the space between the gun tail and the bolt carrier. FIG. 3 indicates the balance block vibration area.

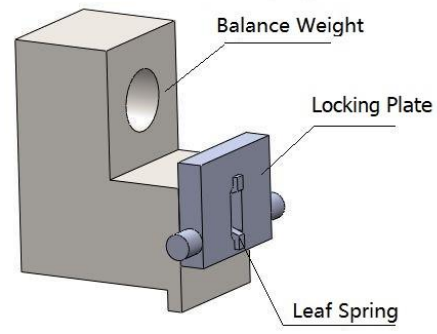

Figure 2. Assembly diagram of balance weight and locking plate

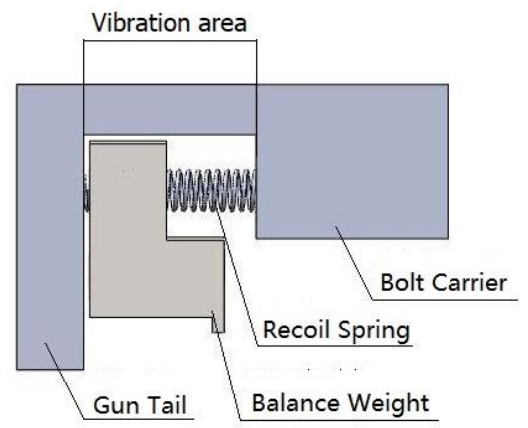

Figure 3. Vibration area of balance weight

\subsection{The principle of balance mechanism}

The bolt carrier and balance weight have their own guide rails. When the bolt carrier recoils, it first contacts the front face of the lock plate and collides with the front face of the lock plate. Because the lock plate and the balance weight are connected together, the lock plate will move back together with the balance weight after being hit by the bolt carrier. During the backward movement of the lock plate, the two cylindrical protrusions on the left and right side walls of the lock plate have been sliding tightly against the lower bottom surface of the release rails. When the slope on the release rail is encountered, the slope forces the lock plate to move downward until it is divorced from the bottom surface of the bolt carrier. Therefore, the interaction between the balance weight and the bolt carrier will have the following situations. Because the volume of the bolt carrier is much larger than the balance block, the mass of the balance block is much smaller than the bolt carrier if the same material is used for both the balance block and the bolt carrier. In fact, in order to ensure the strength of the balance mechanism, the main body of the balance weight can only choose steel, but the balance weight can add heavy metals as a counterweight. When the bolt carrier and the balance weight components collide for the first time, this is a large-mass object hits a small-mass object, and collision separation is easy to occur. But, the stiffness of the balance mechanism spring is much greater than that of the recoil spring. Therefore, when the balance mechanism combination is hit, although it has a high initial speed, it will soon slow down and collide with the bolt carrier again. In this way, before the lock plate being pulled down by the release rail, the collision between the bolt carrier and the balancing mechanism can be regarded as a "collision combination". After the lock plate is forcibly pulled down by the slope on the release rail, if the speed of the bolt carrier is greater than the balance weight, the bolt carrier will pass the position of the lock plate and continue to catch up with the balance weight. Thereafter, if the bolt carrier and balance weight collides, the collision position will occur on the A side of the weight. FIG. 4 is a schematic view of the A side.

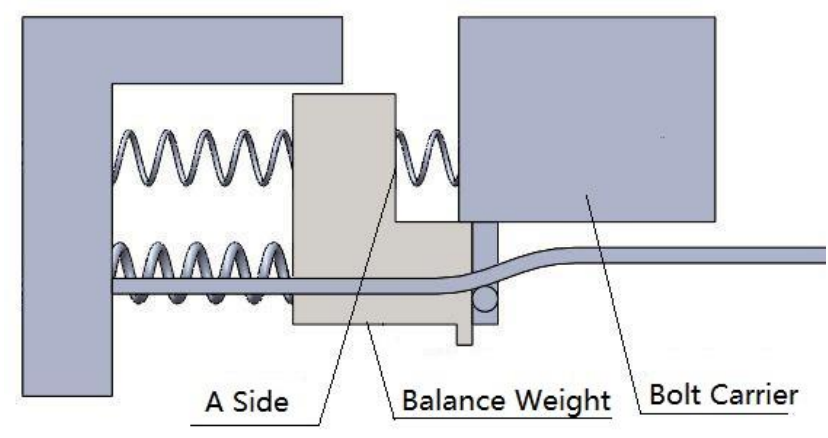

Figure 4. Indication chart of side A of balance weight 


\subsection{Three kinds of mechanism actions that may appear in the balance mechanism system}

When the lock plate is pulled down by the release rail, the bolt carrier can no longer touch the front face of the lock plate, and three situations will occur at this time:

1) Because of the inertia, the balance weight continues to recoil, and constantly compresses the balance spring, and the bolt carrier also continues to recoil. Finally, the bolt carrier moves in the opposite direction because of hitting the gun tail. When the bolt carrier counter-recoils, the balance weight may or may not catch up with the bolt carrier. Finally, the balancing block is blocked by the stop block and cannot move forward. In this case, because the bolt carrier finally hits the tail of the gun, it will still cause a lot of perceptible recoil force, and the balancing mechanism has little effect, so it is inferior.

2) The balance weight continues to recoil due to inertia and constantly compresses the balance mechanism spring, and the bolt carrier also continues to recoil, but because the bolt carrier impacts the balance weight and consumes its own kinetic energy, it will stop recoil due to the lack of kinetic energy when it fails to hit the gun tail, and then starts to move forward under the action of the recoil spring. After the balance block stops moving backward, it also moves forward under the action of the balance mechanism spring, but the balance mechanism is blocked by the block and fails to chase the bolt carrier. This situation leads to the failure of the bolt carrier recoils to its extreme rear position, resulting in the insufficient counter-recoil energy, and in the failure to lock, which is the worst situation and should be avoided;

3 ) The balance weight continues to recoil because of inertia, and constantly compresses the balance mechanism spring, and the bolt carrier also continues to recoil. However, due to the large stiffness of the balance spring, the balance weight quickly decelerates to 0 and moves in the opposite direction. It collides with the recoiling bolt carrier. After the direct collision, the bolt carrier counter-recoils. The balance weight also moves from the muzzle to the tail. This kind of mechanism action causes the bolt carrier to collide with the balance block internally, which causes the bolt carrier to counterrecoils, and avoids the direct collision between the bolt carrier and the gun tail, which produces the perceptible recoil force. Additionally, when the balance block collides with the bolt carrier directly, the kinetic energy of the bolt carrier itself is very limited. The collision not only changes the speed of the bolt carrier, but also increases the counter-recoil kinetic energy of the bolt carrier. This is the best case.

\section{Related dynamics calculation}

When the bolt carrier and the balance weight are in contact for the first time, the bolt carrier has a very high speed, and the balance weight is in a stationary state. At this time, the bolt carrier and the balance weight are in direct impact.

$$
m_{1} v_{1}=m_{1} v_{1}^{\prime}+m_{2} v_{2}^{\prime}
$$

$$
b=\frac{v_{2}^{\prime}-v_{1}^{\prime}}{v_{1}}
$$

In the formula, $m_{1}$ is the mass of the bolt carrier, $m_{2}$ is the mass of the balance mechanism components, $v_{1}$ and $v_{1}^{\prime}$ are the speeds of the bolt carrier before and after impact, $v_{2}^{\prime}$ is the speed of the balance mechanism after impact, $b$ is the recovery factor.

As mentioned above, after a direct impact, the speed of the balance mechanism will soon drop, and then the second and third collisions with the bolt carrier, so the collision between the bolt carrier and the balance mechanism can be regarded as an impact combination ${ }^{[3]}$ :

$$
m_{1} v_{1}=\left(m_{1}+m_{2}\right) v_{2}^{\prime}
$$

where $v_{2}^{\prime}$ is the common speed of the bolt carrier and the balance mechanism after impact combination. The structure dimensions of the balance mechanism are presented in FIG. 5.

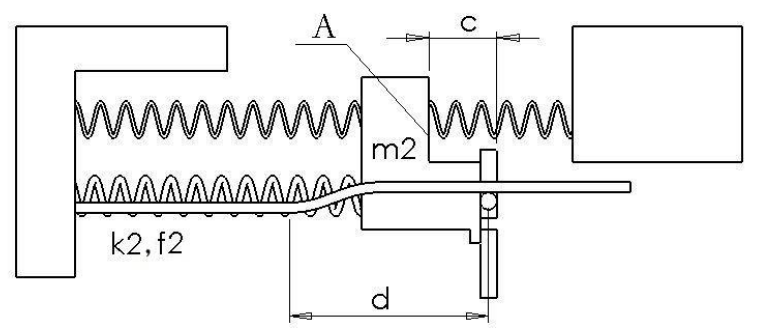

Figure 5. Structure dimensions of balance mechanism

As shown in Figure 5, the horizontal distance between the initial position of the lock plate and the fully released position is set as $d$, the balance weight is $m_{2}$. The distance between the A surface of the balance weight and the front surface of the lock plate is $c$. The spring stiffness of the balance mechanism is $k_{2}$, and the preload is $f_{2}$

The most ideal situation of the balance mechanism is now discussed, that is, the balance block will collide with the bolt carrier, and the collision surface occurs on the A side of the balance block. From the moment when the bolt carrier contacts the lock plate and pushes the balance mechanism backward until the lock plate is pressed down by the release guide rail, and then the bolt carrier collides with face $\mathrm{A}$ on the balance weight, the velocity direction of the bolt carrier does not change, that is, the recoil process is always maintained. Suppose that during the process when the bolt carrier just contacts the front face of the lock plate and collides with the A face of the balance weight, the backward movement distance of bolt carrier is $a$. At this time, although the movement direction of the balance block changes from backward to forward, its displacement is $a-c$. Let the stiffness of the recoil spring be $k_{1}$. When the bolt carrier and the lock plate are just in contact, suppose the spring force of 
the return spring is $F_{N 1}$, the corresponding compression length $h_{1}$ is:

$$
h_{1}=\frac{F_{N 1}}{k_{1}}
$$

Similarly, before the balance mechanism is pushed by the bolt carrier, the spring of the balance mechanism has a pretension $f_{2}$, and also a compression length $h_{2}$ :

$$
h_{2}=\frac{f_{2}}{k_{2}}
$$

Then, from the contact between the bolt carrier and the front face of the lock plate, until the bolt carrier collides with the balance weight on the A surface of the balance weight, the energy absorbed by the recoil spring during this process is:

$$
\begin{aligned}
E_{1} & =\frac{1}{2} k_{1}\left(h_{1}+a\right)^{2}-\frac{1}{2} k_{1} h_{1}^{2} \\
& =\frac{1}{2} k_{1} a\left(2 h_{1}+a\right)
\end{aligned}
$$

Similarly, during this process, the energy absorbed by the balance mechanism spring is:

$$
\begin{aligned}
E_{2} & =\frac{1}{2} k_{2}\left(h_{2}+a\right)^{2}-\frac{1}{2} k_{2} h_{2}^{2} \\
& =\frac{1}{2} k_{2} a\left(2 h_{2}+a\right)
\end{aligned}
$$

Therefore, from the conservation of mechanical energy, it is easy to obtain the kinetic energy and velocity of the balance mechanism and the bolt carrier before collision. Here, the kinetic energy loss due to friction is neglected:

$$
\begin{aligned}
& \frac{1}{2} m_{1} v_{1}^{\prime \prime 2}=\frac{1}{2} m_{1} v_{2}^{\prime 2}-E_{1} \\
& \frac{1}{2} m_{2} v_{2}^{\prime \prime 2}=\frac{1}{2} m_{2} v_{2}^{\prime 2}-E_{2}
\end{aligned}
$$

Set the recoil direction to be positive and list the conservation equation of momentum:

$$
m_{1} v_{1}^{\prime \prime}-m_{2} v_{2}^{\prime \prime}=-m_{1} v_{1}^{\prime \prime \prime}+m_{2} v_{2}^{\prime \prime \prime}
$$

In the formula, $v_{2}^{\prime}$ is the common speed after the bolt carrier collides with the balance mechanism and combines, $v_{1}^{\prime \prime}$ is the speed of the bolt carrier before it collides with the A side of the balance weight, $v_{2}^{\prime \prime}$ is the speed the balance weight has before the A side of the balance weight collides with the bolt carrier, $v_{1}^{\prime \prime \prime}$ is the speed of the bolt carrier after the bolt carrier collides with the A side of the balance weight, $v_{2}^{\prime \prime \prime}$ is the speed of the balance weight after the A face of the balance weight collides with the bolt carrier.

If at this time, $\left|v_{1}^{\prime \prime}\right|>\left|v_{1}^{\prime \prime}\right|$, it means that during the collision between the bolt carrier and the face A of the balance weight, the collision not only reverses the motion direction of the bolt carrier, but also increases its kinetic energy. This is the most ideal state, and also is pursued by the design of the balance mechanism.

\section{Dynamic model}

\subsection{Dynamic model establishment}

First, build a three-dimensional model without a balance mechanism, that is, a full model of the machine gun. In order to simplify the calculation, all parts of the gun are set as rigid bodies. Then import the three-dimensional model into the dynamics software ADAMS to calculate the recoil of the firearm ${ }^{[4]}$. In the next set of tests, a threedimensional model containing a balance mechanism is imported, to calculate the maximum perceptible recoil force during the process before the bolt carrier contacts the balance mechanism until the bolt carrier counterrecoils to its front end position. Compare the calculation results with and without the balance mechanism.

\subsection{Load application}

The machine gun selected in this model is a gas operated machine gun. Because here it does not care about the recoil force caused by gunpowder gas when the firearm is fired, only the pressure of the gas operated chamber is calculated according to Braven empirical formula ${ }^{[3]}$, and then converted into the force added to the bolt carrier. This force drives the bolt carrier to recoil. The other components of the gun are connected to the receiver according to their actual connection modes.

\subsection{Spring parameter setting}

In order to study the recoil reduction effect of the balance mechanism, in the simulation calculations, the same recoil spring is used for the cases with and without balancing mechanism. For the balance mechanism, the pretension of the balance mechanism spring is $80 \mathrm{~N}$. This value is taken to ensure that the balance mechanism will not vibrate back and forth during the process of gun shooting until hitting the balance mechanism. For the stiffness of balance mechanism spring, parametric design is carried out to study the influence of different balance mechanism spring stiffness on the actual effect of sensible recoil.

\subsection{Other parameters setting of balance mechanism}

As mentioned in Section 2 of this article, and refer to FIG. 5 , the parameters in the balance mechanism components are $c, d, m_{2}$, among them, $c, d$ are set according to the space allowed by the machine gun. Because the balance weight can be increased by adding other heavy metal materials, or by increasing the other dimensions except for the size $\mathrm{c}$ to increase the volume, so $m_{2}$ is 
also parameterized, to study the effect of the balance block mass on the reduction of recoil.

\subsection{The setting of sensors in ADAMS}

In recoil simulation, under no chamber pressure applied, there will be four peaks in one cycle of operation of the automaton. The first time is when the pressure of the gas operated device chamber reaches the peak value, and the second time is when the guide post of the bolt carrier enters into the curve groove of belt feed lever, and the third and fourth time is when the bolt carrier hits the gun tail or balance mechanism respectively. If there is no balance mechanism structure, there will be 3 peaks of recoil. Because the first two times have nothing to do with this study, the balance mechanism can not reduce the recoil force caused by the pressure of the gas operated device, nor can it reduce the recoil force caused by the impact between the bolt carrier and the feed mechanism, the Adams sensor technology is used to measures the peak value of the third and fourth times of recoil force, and the first two peak recoil forces don't record.

\subsection{Recoil curve of a machine gun without balance mechanism}

In the simulation, taking a small caliber machine gun as an example, there is no restriction between the whole machine gun and the ground, and there is no gravity in the simulation environment. The third peak in the automaton cycle is calculated, that is, the recoil curve produced by the collision between the bolt carrier and the gun tail. FIG. 6 shows sensible recoil curve without balance mechanism, and FIG. 7 presents the speed curve of bolt carrier without balance mechanism.

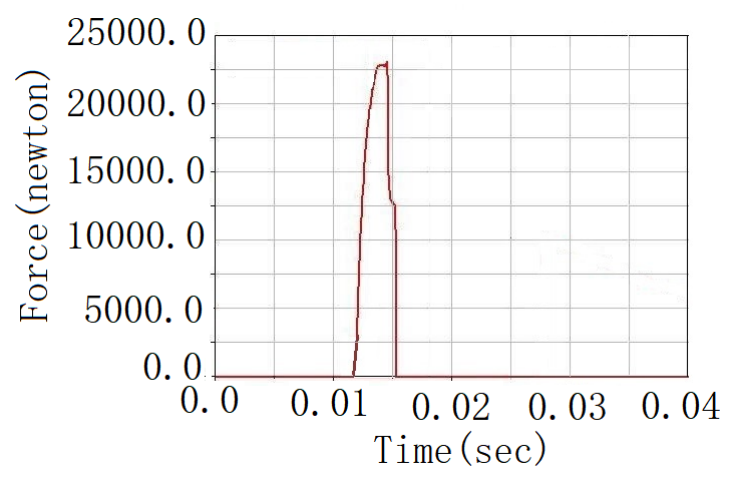

Figure 6. sensible recoil curve without balance mechanism

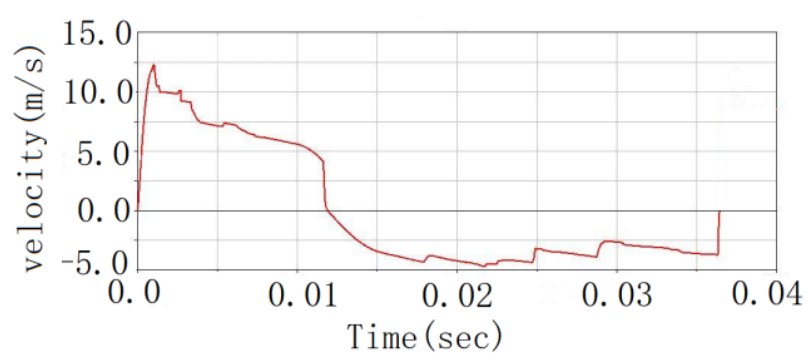

Figure 7. speed curve of bolt carrier without balance mechanism

As shown in FIG. 6, the peak value represents the perceptible recoil force generated when the bolt carrier and gun tail collide directly. Under the condition without balancing mechanism, the maximum recoil force is 22615N. As shown in FIG. 7, at this time, it takes about 0.037 second for the machine gun to complete an automatic cycle.

\subsection{Parametric simulation of machine gun with balance mechanism components}

As described in sections 3.3 and 3.4 above, the spring stiffness and balance weight mass in the balance mechanism components are parameterized. Because it is difficult to change the shape of balance block in simulation, the parameterization of balance block mass can be changed to the parameterization of its density. As the bolt carrier is made of alloy steel with a density of $7800 \mathrm{~kg} / \mathrm{m}^{3}$, the density of the balance block is parameterized from 0.5 to 2 times of its density, that is, the density is taken as $3900 \mathrm{~kg} / \mathrm{m}^{3}$ to $15600 \mathrm{~kg} / \mathrm{m}^{3}$. It can be divided into four uniform sections, and 5 values are used for research. In this paper, the stiffness of the recoil spring of the machine gun is $1400 \mathrm{~N} / \mathrm{m}$. Because the spring stiffness of the balance mechanism must be greater than that of the recoil spring, the spring stiffness of the balance mechanism shall be taken as $2000 \mathrm{~N} / \mathrm{m}$ to $5000 \mathrm{~N} / \mathrm{m}$. According to the simulation analysis, when the spring stiffness of balance mechanism is more than $5500 \mathrm{~N} / \mathrm{m}$, the second situation described in Section 1.3 is easy to occur, that is, the recoil energy of the bolt carrier is insufficient, so the maximum spring stiffness of the balance mechanism is $5000 \mathrm{~N} / \mathrm{m}$. In addition to the first and last values of 2000 and 5000, three values are evenly selected in the middle, and a total of five values are summed up. In this way, the density and stiffness are 25 groups of values. Call the iSIGHT module of Adams for simulation analysis ${ }^{[4]}$, the simulation results are shown in FIG. 8 and Table 1. 


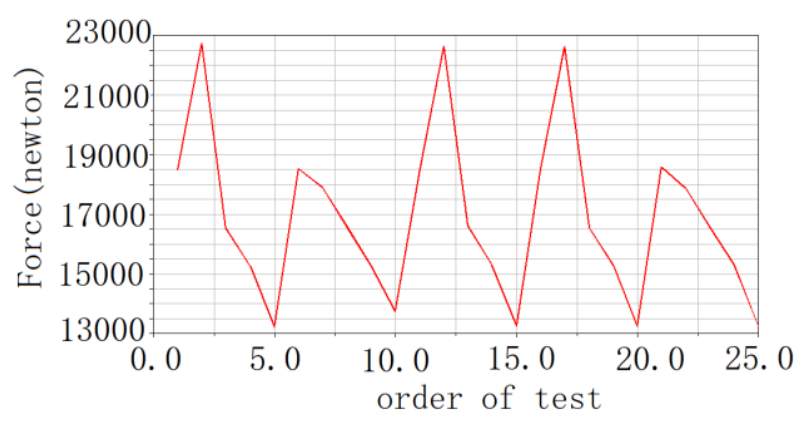

Figure 8. Adams parametric design result chart

As shown in FIG. 8 and Table 1, the perceptible recoil in several groups are greater than $22000 N$, and the maximum perceptible recoil is $22744 N$, near to the value $22615 N$ for the case without balancing mechanism, that is, the balance mechanism does not work. Through analysis, it is found that, in this case, although there is no case that the bolt carrier extrudes the balancer and the balance weight further hits the gun tail at the same time, the bolt carrier and the balancer almost hit the gun tail at the same time. From the perspective of momentum conservation, even if the balance mechanism component is added, the bolt carrier shares part of the momentum to the balance mechanism, but there is no external force supplement in this process, so the total momentum will not increase. However, because the stiffness of the balance mechanism spring is higher than that of the recoil spring, the perceptible recoil is higher after adding the balance mechanism due to the influence of the stiffness of the balance mechanism spring.

However, it can be seen from FIG. 8 that after using the balance mechanism, if the parameters of the balance mechanism are proper, the sensed recoil force can be reduced to $13234 N, 41.5 \%$ less than $22615^{N}$ for the case without balance mechanism.

Table 1. Parameterized results of recoil force

\begin{tabular}{|c|c|c|c|}
\hline $\begin{array}{c}\text { Order } \\
\text { of test }\end{array}$ & $\begin{array}{c}\text { Spring stiffness } \\
\text { of balance } \\
\text { mechanism } \\
(N / m)\end{array}$ & $\begin{array}{c}\text { Balance weight } \\
\text { density } \\
\left(k g / m^{3}\right.\end{array}$ & $\begin{array}{c}\text { Recoil } \\
\text { force } \\
(N)\end{array}$ \\
\hline 1 & 2000.0 & 3900 & 18501 \\
\hline 2 & 2000.0 & 6825 & 22744 \\
\hline 3 & 2000.0 & 9750 & 16531 \\
\hline 4 & 2000.0 & 12675 & 15261 \\
\hline 5 & 2000.0 & 15600 & 13234 \\
\hline 6 & 2750 & 3900 & 18524 \\
\hline 7 & 2750 & 6825 & 17891 \\
\hline 8 & 2750 & 9750 & 16577 \\
\hline 9 & 2750 & 12675 & 15264 \\
\hline 10 & 2750 & 15600 & 13749 \\
\hline 11 & 3500 & 3900 & 18500 \\
\hline 12 & 3500 & 6825 & 22640 \\
\hline 13 & 3500 & 9750 & 16612 \\
\hline 14 & 3500 & 12675 & 15298 \\
\hline 15 & 3500 & 15600 & 13262 \\
\hline 16 & 4250 & 3900 & 18539 \\
\hline 17 & 4250 & 6825 & 22631 \\
\hline 18 & 4250 & 9750 & 16572 \\
\hline 19 & 4250 & 12675 & 15304 \\
\hline & & & \\
\hline
\end{tabular}

\begin{tabular}{|c|c|c|c|}
\hline 20 & 4250 & 15600 & 13250 \\
\hline 21 & 5000.0 & 3900 & 18576 \\
\hline 22 & $\mathbf{5 0 0 0 . 0}$ & 6825 & 17876 \\
\hline 23 & 5000.0 & 9750 & 16561 \\
\hline 24 & $\mathbf{5 0 0 0 . 0}$ & 12675 & 15326 \\
\hline 25 & $\mathbf{5 0 0 0 . 0}$ & 15600 & 13252 \\
\hline
\end{tabular}

It can be seen from Table 1 that the main factor influencing the perceptible recoil force is the mass of the balance weight. Within the allowable stiffness range of the balance mechanism spring, its stiffness has little influence on the reduction of recoil effect of the balance mechanism.

\subsection{Simulation verification}

Now take order 5 in Table 1, the group of optimal values for simulation verification. The spring stiffness of the balance mechanism is $2000 \mathrm{~N} / \mathrm{m}$, and the density of balance block is $15600 \mathrm{~kg} / \mathrm{m}^{3}$. The recoil force obtained by simulation is $13234 N$. The simulated curves of recoil force, balancer speed and bolt carrier speed are shown in FIG. 9 to FIG. 11. As the reason mentioned in section 3.5, the first two recoils are not included in FIG. 9.

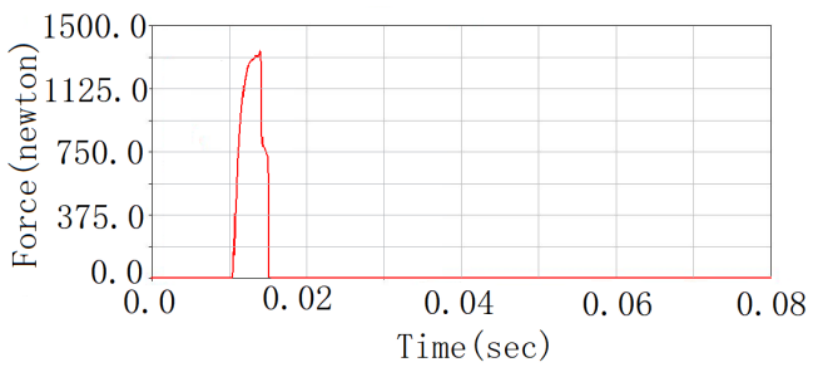

Figure 9. Curve of recoil force

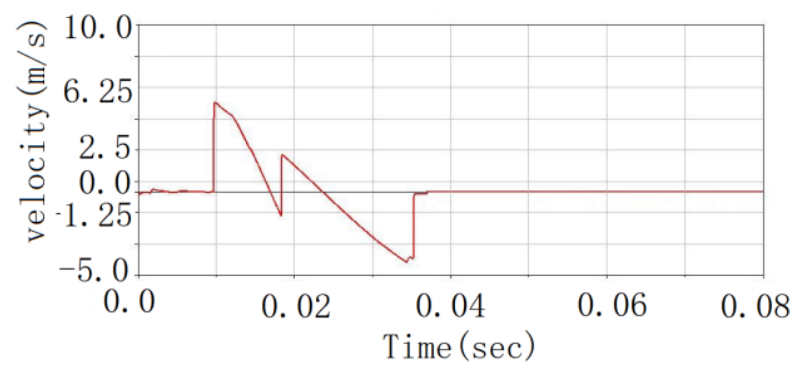

Figure 10. Curve of balancer speed

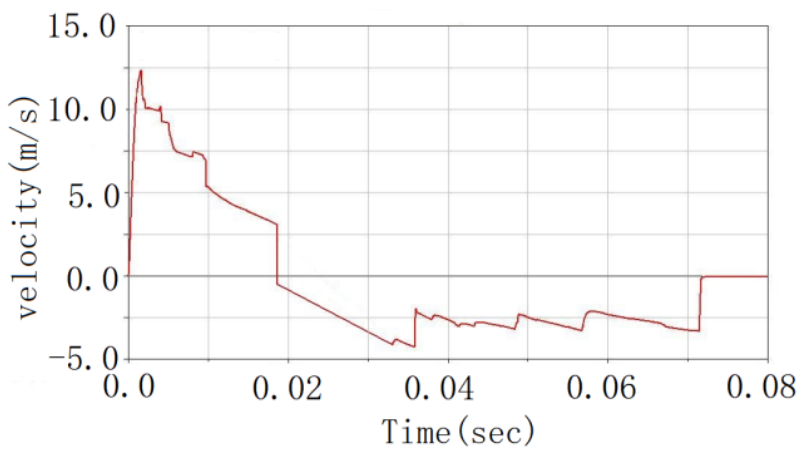

Figure 11. Curve of bolt carrier speed 
As shown in FIG. 9, at about 0.01 second, the bolt carrier collides with the balance weight, and the speed of the balance weight suddenly increases. In ADAMS, the recoil direction is the positive direction of velocity, and this is also the time when the gun produces the maximum recoil force. After that, both the balancer and the bolt carrier start to decelerate under the action of their own springs. The balancer's speed quickly reduces to 0 , then accelerates in reverse direction, and collides with the bolt carrier again at about 0.018 second. In this collision, the speed of the bolt carrier is forced to reverse, and the velocity of the balance mechanism itself is also reversed, that is, the two components collide and separate. At 0.018 second, when the bolt carrier collides with the balancer, almost no perceptible recoil, which realizes the original purpose of the balancer design, that is to say, the collision between the bolt carrier and the gun tail becomes the internal collision between the bolt carrier and the balance mechanism, and the recoil force of the shooter is eliminated. As mentioned above, when there is no balance mechanism, one automatic cycle time is $0.037 \mathrm{~s}$. After adding the balance mechanism, the energy of the bolt carrier is lost when it counter-recoils, so the counterrecoil time of the bolt carrier is longer. Finally, it collides with the barrel extension at about 0.072 seconds to complete the counter-recoil of the bolt carrier.

\section{Conclusions}

In this paper, an internal impact balance mechanism is designed to reduce the recoil force of the machine gun. Through parametric analysis, the influence of balance mechanism spring and balance mechanism mass on the effect of recoil reduction is explored. Through Adams parametric simulation, the better results are acquired, and the following conclusions are obtained:

1) According to the results of parametric analysis, the influence of balance mechanism mass on recoil reduction is greater than that of balance mechanism spring within their own allowable ranges.

2) For the most ideal design of the balance mechanism, the generation of recoil force mainly comes from the first direct collision between the moving bolt carrier and the stationary balance mechanism, while the second collision between the balance mechanism and the bolt carrier has the opposite velocities, which hardly produces perceptible recoil.

At present, this paper only conducts a theoretical analysis and simulation study on the feasibility of reducing the perceptible recoil of a machine gun with an internal impact balancing mechanism. The specific effect of reducing the perceptible recoil is affected by the spring stiffness of the balance mechanism, the mass of the balancing block, and the relevant geometric parameters. Through further parameter optimization, an ideal effect of reducing the recoil can be obtained.

\section{References}

1. HE Dawei, LI Zhongxin. Analysis of Spring Buffer Influence on a Gun' s RF and Recoil. J. Ordnance Industry Automation, 34(01), 44-48+55 (2015)

2. SONG Jie. Study on Low Recoil Launching Technology of Grenade Machine Gun System. (Nanjing University of Science and Technology, 2017)

3. WANG Yaping, XU Chen, WANG Yongjuan, YAO Yangwu. Dynamics of Artillery and Automatic Weapons (Beijing: BEIJING INSTITUTE OF TECHNOLOGY PRESS, 2014)

4. GONG Penghan, HU Renxi, KANG Shiting. ADAMS virtual prototype 2014 from entry to mastery (Beijing: CHINA MACHINE PRESS, 2016)

5. Xiao Junbo, Yang Guolai, Li Hongqiang, Qiu Ming, Liao Zhenqiang. Influence of Matching Muzzle Brake and Buffer on Weapon Recoil. J. Journal of Ballistics, 29(04), 86-92 (2017)

6. Hua Hongliang, Liao Zhengqiang, Zhang Xiangyan,.Muzzle dynamic characteristics analysis and its matching for firing accuracy improvement. J. Journal of Vibration and Shock, 36(08),29-33(2017)

7. Lu Ye, Zhou Kedong, He Lei, Li Junsong, Huang Xueying. Research on Influence of Muzzle Brake Efficiency on a New Large Caliber Machina Gun Based on Floating Principle. J. ACTA ARMAMENTARII,27(09),1585-1591(2016) 